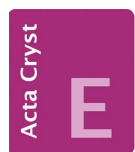

CRYSTALLOGRAPHIC COMMUNICATIONS

ISSN 2056-9890

Received 5 March 2016

Accepted 18 March 2016

Edited by C. Rizzoli, Universita degli Studi di Parma, Italy

Keywords: crystal structure; 2-aminoanilinium; hydrogen phosphate; supramolecular network; hydrogen bonds.

CCDC reference: 1469440

Supporting information: this article has supporting information at journals.iucr.org/e

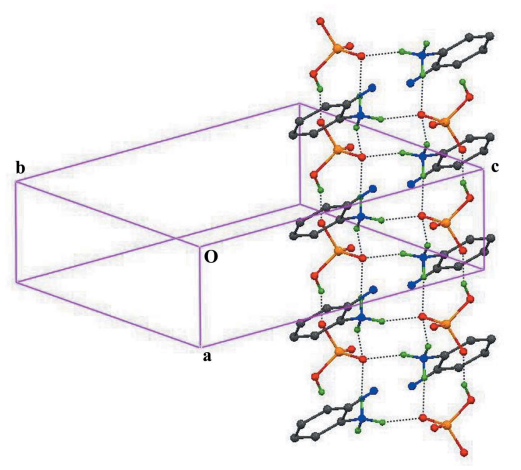

\section{Crystal structure of bis(2-aminoanilinium) hydrogen phosphate}

\author{
Reena Ittyachan, ${ }^{a}$ Melesuparambil Sundaram Ahigna ${ }^{a}$ and Rajamony Jagan ${ }^{b *}$ \\ ${ }^{\mathbf{a}}$ Department of Physics, Sacred Heart College, Chalakudy, Kerala 680 307, India, and ${ }^{\mathbf{b}}$ Department of Chemistry, Indian \\ Institute of Technology Madras, Chennai 600 036, India. *Correspondence e-mail: phyjagan@gmail.com
}

The asymmetric unit of the title compound, $2 \mathrm{C}_{6} \mathrm{H}_{9} \mathrm{~N}_{2}{ }^{+} \cdot \mathrm{HPO}_{4}{ }^{2-}$, comprises two 2-aminoanilinium cations and one hydrogen phosphate dianion. In the crystal, the $\mathrm{HPO}_{4}{ }^{2-}$ dianions are linked by $\mathrm{O}-\mathrm{H} \cdots \mathrm{O}$ hydrogen bonds into chains along [100]. The inorganic anionic chains and organic cations are linked by $\mathrm{N}-\mathrm{H} \cdots \mathrm{O}$ and $\mathrm{N}-\mathrm{H} \cdots \mathrm{N}$ hydrogen bonds, forming a two-dimensional supramolecular network extending parallel to (001).

\section{Chemical context}

Inorganic-organic hybrid compounds are of current interest due to their fascinating architectures and potential applications in crystal engineering and supramolecular chemistry (Singh et al., 2011; Direm et al., 2015). Among the explored hybrid compounds, organic phosphates formed as a result of the reaction with inorganic oxy acids such as orthophosphoric acid $\left(\mathrm{H}_{3} \mathrm{PO}_{4}\right)$ and organic amines and amides are particularly interesting. Organic monohydrogen $\left(\mathrm{HPO}_{4}{ }^{2-}\right)$ and dihydrogen phosphate $\left(\mathrm{H}_{2} \mathrm{PO}_{4}^{-}\right)$compounds provide a class of materials with numerous practical and potential uses in various fields such as biomolecular sciences, catalysis, liquidcrystal-material development, ferroelectrics, non-linear optical and supramolecular studies (Khan et al., 2009; Evans et al., 2008; Balamurugan et al., 2010). Non-covalent interactions, such as hydrogen bonding and other weak interactions, represent the basic set of tools for the construction of elaborate supramolecular architectures of organic or inorganic-organic compounds. In this respect, the potential of monohydrogen and dihydrogen phosphate anions as useful building blocks has been investigated structurally (Shylaja et al., 2008; Oueslati et al., 2007; Jagan et al., 2015; Trojette et al., 1998; Soumhi \& Jouini, 1995). Here we report the structure and the self-assembled supramolecular architecture exhibited through the formation of $\mathrm{O}-\mathrm{H} \cdots \mathrm{O}, \mathrm{N}-\mathrm{H} \cdots \mathrm{O}$ and $\mathrm{N}-$ $\mathrm{H} \cdots \mathrm{N}$ hydrogen bonds in bis(2-aminoanilinium) hydrogen phosphate.<smiles>Nc1ccccc1N</smiles> 


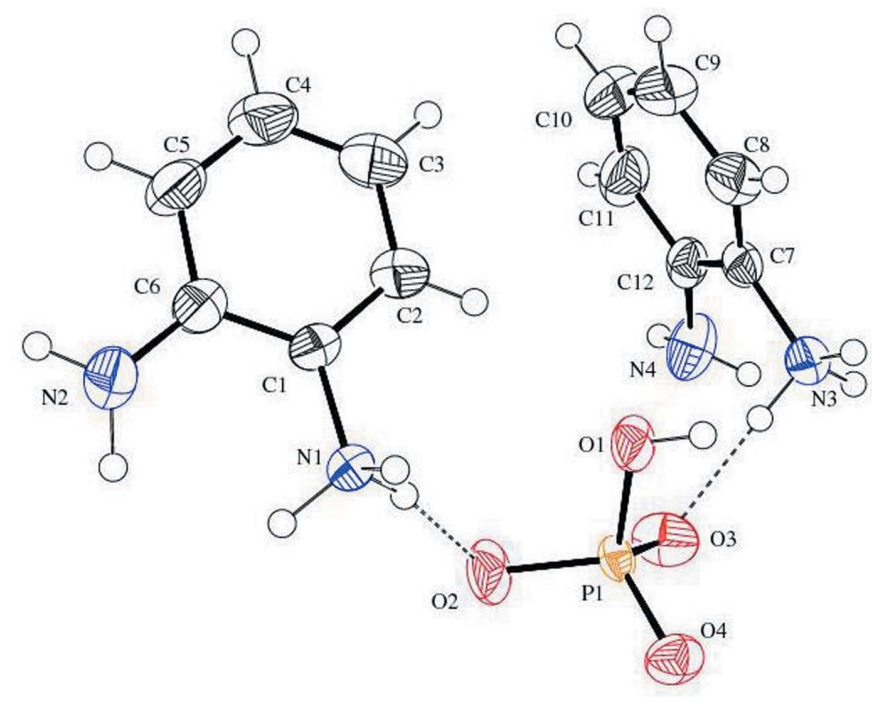

Figure 1

The asymmetric unit of the title compound with displacement ellipsoid drawn at the $40 \%$ probability level. The dashed lines represent hydrogen bonds.

\section{Structural commentary}

The asymmetric unit of the title compound comprises two 2-aminoanilinium cations and one hydrogen phosphate dianion (Fig. 1). The existence of the hydrogen phosphate anion is confirmed by the $\mathrm{P}-\mathrm{O}$ bond distances, and the presence of a relevant density peak at a distance from the oxygen atom $\mathrm{O} 1$ confirms the hydroxyl group of the anion. The bond distance $\mathrm{P} 1-\mathrm{O} 1=1.561$ (2) $\AA$ indicates single-bond character, while the bond distances $\mathrm{P} 1-\mathrm{O} 2=1.504$ (2), $\mathrm{P} 1-$ $\mathrm{O} 3=1.504$ (2) and $\mathrm{P} 1-\mathrm{O} 4=1.497$ (2) $\AA$ reveal the resonating $\mathrm{P}-\mathrm{O}$ bonds of the hydrogen phosphate anion. As expected (Rao et al., 2010; Peng \& Zhao, 2010), in both cations the C$\mathrm{N}$ bond $[\mathrm{C} 1-\mathrm{N} 1=1.450(3), \mathrm{C} 7-\mathrm{N} 3=1.450$ (4) $\AA$ ] involving the ammonium group is longer than that in the amine group $[\mathrm{C} 6-\mathrm{N} 2=1.384(4), \mathrm{C} 12-\mathrm{N} 4=1.383(4) \AA]$. The phenyl rings of the $o$-phenylenediammonium cations are almost perpendicular to one another [dihedral angle $86.53(2)^{\circ}$ ].

\section{Supramolecular features}

In the title structure, the hydrogen phosphate anion and 2-aminoanilinium cations possess a number of donor and acceptor sites, which leads to the formation of a variety of hydrogen bonds viz. $\mathrm{O}-\mathrm{H} \cdots \mathrm{O}, \mathrm{N}-\mathrm{H} \cdots \mathrm{O}$ and $\mathrm{N}-\mathrm{H} \cdots \mathrm{N}$ (Table 1). The $\mathrm{O} 1-\mathrm{H} 1 D \cdots \mathrm{O} 2^{\mathrm{i}}$ hydrogen bond [symmetry code: (i) $x+1, y, z]$ connects adjacent hydrogen phosphate anions, forming anionic chains extending along [100]. The oxygen atom $\mathrm{O} 3$ acts as a trifurcated hydrogen-bond acceptor for the donor nitrogen atom $\mathrm{N} 1$ at $(x, y, z),(-1+x, y, z)$ and $(1-x, 1-y, 2-z)$, forming a one-dimensional supramolecular ladder extending along [100] as shown in Fig. 2. In the ladder, centrosymmetrically related anions and cations are interlinked through $\mathrm{N} 3-\mathrm{H} 3 C \cdots \mathrm{O} 3, \mathrm{~N} 3-\mathrm{H} 3 A \cdots \mathrm{O} 3^{\mathrm{i}}$ and $\mathrm{N} 3-\mathrm{H} 3 B \cdots \mathrm{O}^{\text {iv }}$ [symmetry code: (iv) $-x+1,-y+1,-z+2$ ]
Table 1

Hydrogen-bond geometry $\left(\AA,^{\circ}\right)$.

\begin{tabular}{lllll}
\hline$D-\mathrm{H} \cdots A$ & $D-\mathrm{H}$ & $\mathrm{H} \cdots A$ & $D \cdots A$ & $D-\mathrm{H} \cdots A$ \\
\hline $\mathrm{O} 1-\mathrm{H} 1 D \cdots \mathrm{O} 2^{\mathrm{i}}$ & $0.85(1)$ & $1.65(1)$ & $2.470(3)$ & $164(4)$ \\
$\mathrm{N} 3-\mathrm{H} 3 A \cdots 3^{\mathrm{i}}$ & $0.90(2)$ & $2.06(2)$ & $2.928(3)$ & $160(3)$ \\
$\mathrm{N} 1-\mathrm{H} 1 A \cdots \mathrm{O} 4^{\mathrm{ii}}$ & $0.92(2)$ & $1.81(2)$ & $2.720(3)$ & $171(3)$ \\
$\mathrm{N} 1-\mathrm{H} 1 C \cdots \mathrm{O} 4^{\mathrm{iii}}$ & $0.93(2)$ & $2.02(2)$ & $2.953(3)$ & $179(3)$ \\
$\mathrm{N} 2-\mathrm{H} 2 A \cdots \mathrm{O} 4^{\mathrm{iii}}$ & $0.92(2)$ & $1.99(2)$ & $2.904(4)$ & $170(3)$ \\
$\mathrm{N} 4-\mathrm{H} 4 A \cdots \mathrm{O} 4^{\mathrm{i}}$ & $0.88(2)$ & $2.45(3)$ & $3.188(4)$ & $142(3)$ \\
$\mathrm{N} 3-\mathrm{H} 3 B \cdots \mathrm{O} 3^{\text {iv }}$ & $0.91(2)$ & $1.87(2)$ & $2.740(3)$ & $159(3)$ \\
$\mathrm{N} 3-\mathrm{H} 3 C \cdots \mathrm{O} 3$ & $0.91(2)$ & $1.87(2)$ & $2.778(3)$ & $176(3)$ \\
$\mathrm{N} 1-\mathrm{H} 1 B \cdots \mathrm{O} 2$ & $0.92(2)$ & $1.83(2)$ & $2.734(3)$ & $169(3)$ \\
$\mathrm{N} 4-\mathrm{H} 4 B \cdots \mathrm{N}^{\mathrm{v}}$ & $0.89(2)$ & $2.33(2)$ & $3.210(4)$ & $172(3)$ \\
\hline
\end{tabular}

Symmetry codes: (i) $x+1, y, z$; (ii) $-x+1,-y+2,-z+2$; (iii) $-x,-y+2,-z+2$; (iv) $-x+1,-y+1,-z+2$; (v) $x, y-1, z$.

hydrogen bonds, forming two types of fused rings of $R_{4}^{2}(8)$ graph-set motif. The association of $\mathrm{O}-\mathrm{H} \cdots \mathrm{O}$ hydrogen bonds in the anionic chains with the $\mathrm{N}-\mathrm{H} \cdots \mathrm{O}$ hydrogen bonds in the ladder forms heteromeric $R_{3}^{3}(10)$ hydrogen-bonded motifs. Adjacent ladders are further bridged by $\mathrm{N} 1-\mathrm{H} 1 B \cdots \mathrm{O} 2$, $\mathrm{N} 1-\mathrm{H} 1 A \cdots \mathrm{O} 4^{\mathrm{ii}}$ and $\mathrm{N} 1-\mathrm{H} 1 C \cdots \mathrm{O} 4^{\mathrm{iii}}$ [symmetry codes: (ii) $-x+1,-y+2,-z+2$; (iii) $-x,-y+2,-z+2]$ hydrogen bonds, resulting in the formation of a two-dimensional organic-inorganic supramolecular layered network parallel to (001) (Fig. 3). In the (001) network, the bridging cations make rings of $R_{3}^{3}(10)$ and $R_{5}^{3}(12)$ motifs through the three chargeassisted $\mathrm{N}-\mathrm{H} \cdots \mathrm{O}$ and the $\mathrm{O} 1-\mathrm{H} 1 D \cdots \mathrm{O} 2^{\mathrm{i}}$ hydrogen bonds. In addition, the $\mathrm{N} 2-\mathrm{H} 2 A \cdots \mathrm{O} 4^{\mathrm{iii}}, \mathrm{N} 1-\mathrm{H} 1 C \cdots \mathrm{O} 4^{\mathrm{iii}}$ and $\mathrm{N} 4-$ $\mathrm{H} 4 B \cdots \mathrm{N} 2^{\mathrm{v}}$ [symmetry codes: (iii) $-x,-y+2,-z+2$; (v) $x,-1+y, z]$ hydrogen bonds stabilize the (001) network. In

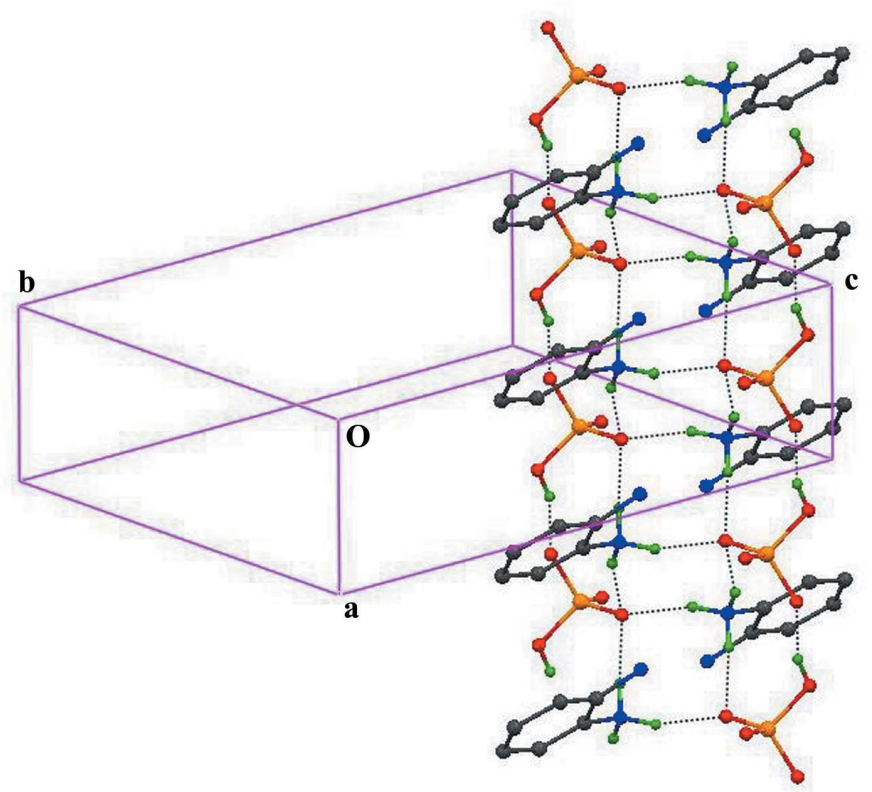

Figure 2

Partial packing diagram of the title compound showing the formation of an organic-inorganic supramolecular ladder through $\mathrm{N}-\mathrm{H} \cdots \mathrm{O}$ and $\mathrm{O}-$ $\mathrm{H} \cdots \mathrm{O}$ hydrogen bonds extending along [100]. The formation of rings with $R_{4}^{2}(8)$ and $R_{3}^{3}(10)$ graph-set motifs is also shown. Hydrogen atoms not involved in hydrogen bonding are omitted for clarity. 


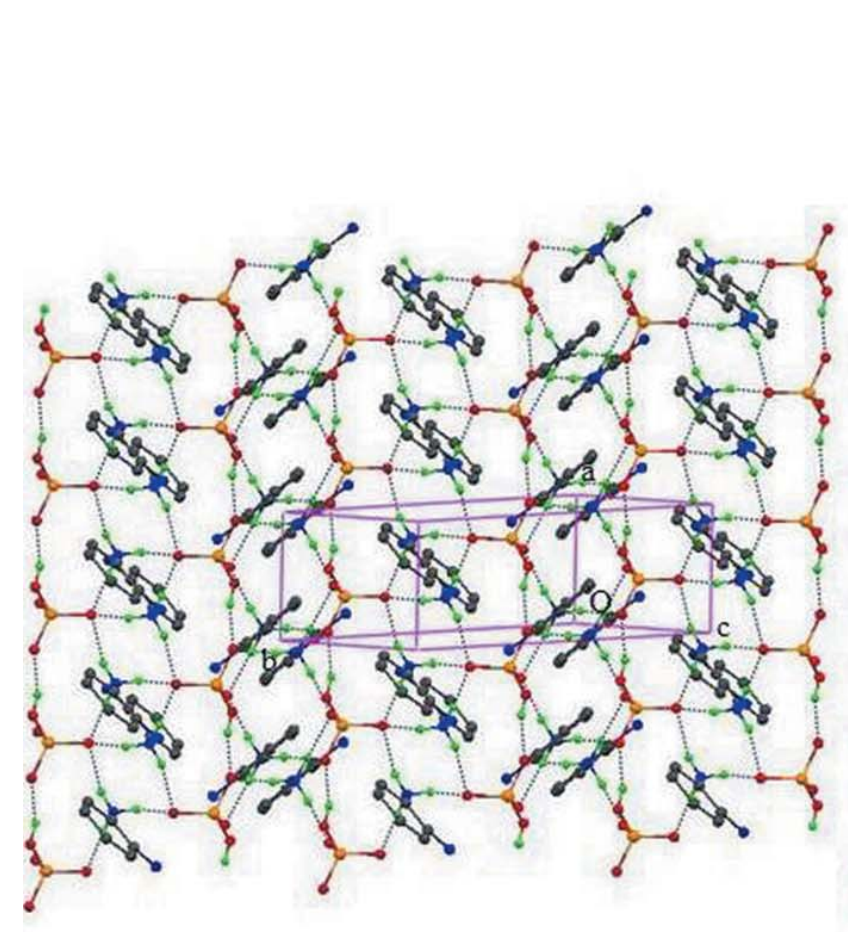

(a)

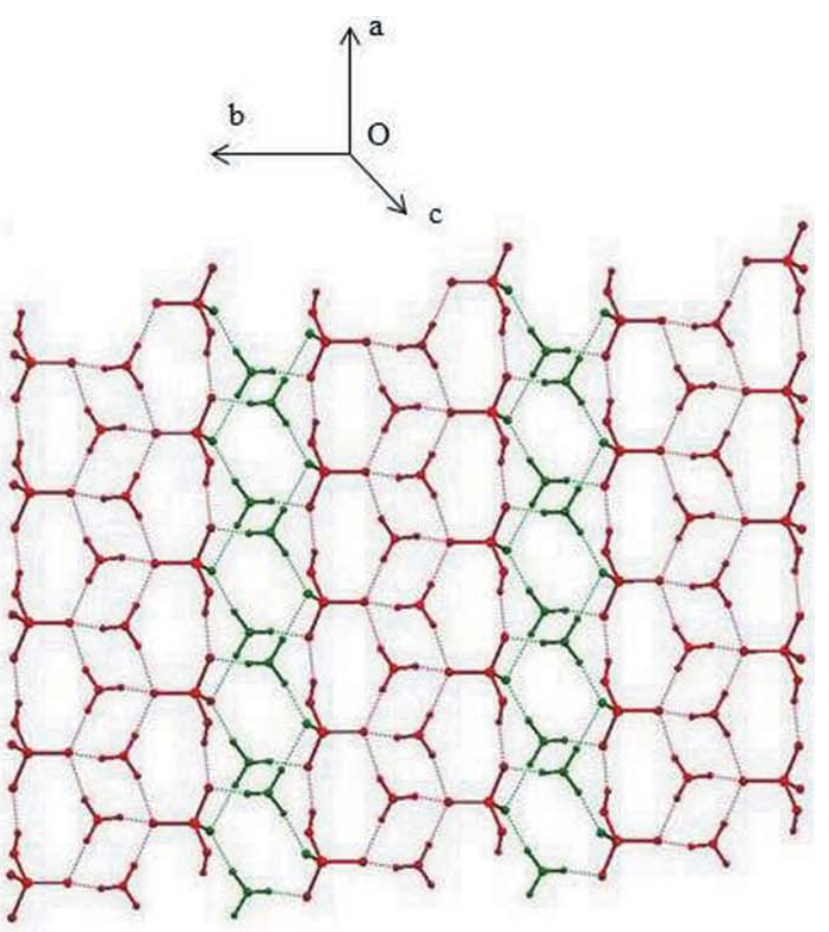

(b)

Figure 3

Crystal packing of the title compound showing (a) the formation through hydrogen bonds (dashed lines) of an organic-inorganic supramolecular sheet extending parallel to (001) and $(b)$ the (001) network in which red represents the [100] ladder, bridged by the cations (represented in green) through $\mathrm{N}-$ $\mathrm{H}$... O hydrogen bonds.

the crystal structure (Fig. 4), adjacent organic-inorganic layers are separated by a distance equal to the length of the $c$ axis.

\section{Database Survey}

A CSD database search (ConQuest 1.17; Groom \& Allen, 2014) showed 48 entries for hydrogen phosphate salts formed with various amino cations. It is interesting to observe that most of the reported structures of hydrogen phosphate salts are hydrated (33 structures) compared to the reported structures of dihydrogen phosphate and phosphate salts. Most of the hydrogen phosphate structures reported contain alkyl cations (Ilioudis et al., 2002; Mrad et al., 2012; Li et al., 2010), in which the alkyl cations encapsulated between chains of hydrogen phosphate are flexible with respect to the nature of the cations, which may induce a change in physical properties (Baouab \& Jouini, 1998). As observed in the title compound, in the crystal structure of 2-aminoanilinium dihydrogen phosphate (CSD refcode: SAYWAQ; Trojette et al., 1998), the dihydrogen phosphate anions form chains, which are bridged by 2-aminoanilinium cations through $\mathrm{N}-\mathrm{H} \cdots \mathrm{O}$ hydrogen bonds, generating a two-dimensional inorganic-organic network. Conversely, in the crystal structure of 1,2-phenylenediammonium bis(dihydrogen phosphate) (ZAYPAQ; Soumhi \& Jouini, 1995), the anions form inorganic sheets interlinked by 1,2-phenylenediammonium cations, thus generating a three-dimensional inorganic-organic framework.
This can be attributed to the double protonation of the cations in ZAYPAQ compared to the title compound and SAYWAQ. In the crystal structure of 2-aminoanilinium perchlorate monohydrate (KAJGUY; Raghavaiah et al., 2005), the 2-aminoanilinium cation, the perchlorate anion and the lattice water molecule assemble into a unique hydrogen-bonded supramolecular framework, forming alternate hydrophobic and hydrophilic zones.

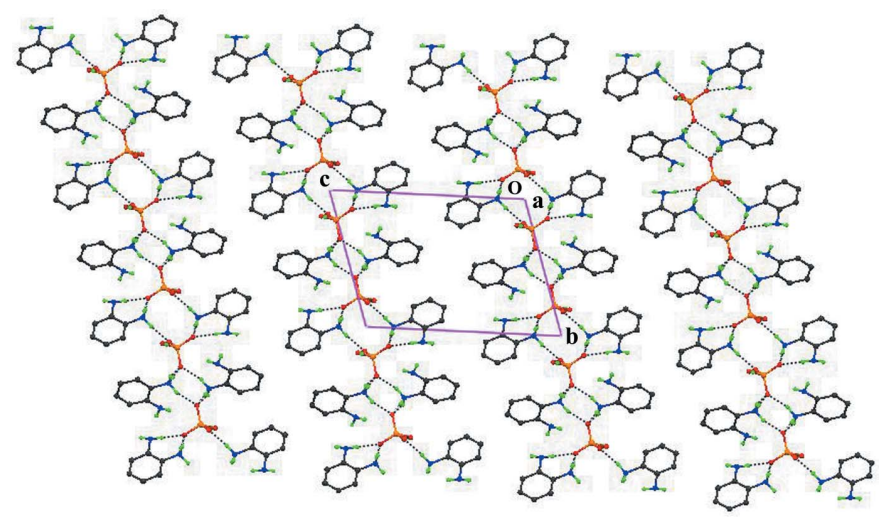

Figure 4

Packing of the title compound, viewed down the $a$ axis, showing the arrangement of the (001) two-dimensional supramolecular networks stacked along the $c$ axis. Dashed lines indicate hydrogen bonds. 
Table 2

Experimental details.

\begin{tabular}{|c|c|}
\hline \multicolumn{2}{|l|}{ Crystal data } \\
\hline Chemical formula & $2 \mathrm{C}_{6} \mathrm{H}_{9} \mathrm{~N}_{2}{ }^{+} \cdot \mathrm{HPO}_{4}{ }^{2-}$ \\
\hline$M_{\mathrm{r}}$ & 314.28 \\
\hline Crystal system, space group & Triclinic, $P \overline{1}$ \\
\hline Temperature $(\mathrm{K})$ & 296 \\
\hline$a, b, c(\AA)$ & $4.7613(7), 10.8925(17), 15.054(2)$ \\
\hline$\alpha, \beta, \gamma\left({ }^{\circ}\right)$ & $107.263(3), 94.060(3), 94.549(3)$ \\
\hline$V\left(\AA^{3}\right)$ & $739.6(2)$ \\
\hline$Z$ & 2 \\
\hline Radiation type & Мo $K \alpha$ \\
\hline$\mu\left(\mathrm{mm}^{-1}\right)$ & 0.21 \\
\hline Crystal size $(\mathrm{mm})$ & $0.30 \times 0.20 \times 0.20$ \\
\hline \multicolumn{2}{|l|}{ Data collection } \\
\hline Diffractometer & $\begin{array}{l}\text { Bruker Kappa APEXII CCD } \\
\text { Diffractometer }\end{array}$ \\
\hline Absorption correction & $\begin{array}{l}\text { Multi-scan (SADABS; Bruker, } \\
\text { 2012) }\end{array}$ \\
\hline$T_{\min }, T_{\max }$ & $0.865,0.902$ \\
\hline $\begin{array}{l}\text { No. of measured, independent and } \\
\text { observed }[I>2 \sigma(I)] \text { reflections }\end{array}$ & $16948,2841,2271$ \\
\hline$R_{\text {int }}$ & 0.039 \\
\hline$(\sin \theta / \lambda)_{\max }\left(\AA^{-1}\right)$ & 0.617 \\
\hline \multicolumn{2}{|l|}{ Refinement } \\
\hline$R\left[F^{2}>2 \sigma\left(F^{2}\right)\right], w R\left(F^{2}\right), S$ & $0.052,0.119,1.16$ \\
\hline No. of reflections & 2841 \\
\hline No. of parameters & 242 \\
\hline No. of restraints & 11 \\
\hline $\mathrm{H}$-atom treatment & $\begin{array}{l}\mathrm{H} \text { atoms treated by a mixture of } \\
\text { independent and constrained } \\
\text { refinement }\end{array}$ \\
\hline$\Delta \rho_{\max }, \Delta \rho_{\min }\left(\mathrm{e} \AA^{-3}\right)$ & $0.49,-0.34$ \\
\hline
\end{tabular}

Computer programs: APEX2, SAINT and XPREP (Bruker, 2012), SHELXS2014 (Sheldrick, 2008), SHELXL2014 (Sheldrick, 2015), ORTEP-3 for Windows (Farrugia, 2012), Mercury (Macrae et al., 2008) and PLATON (Spek, 2009).

\section{Synthesis and crystallization}

The title compound was prepared by dissolving in water $o$-phenylenediamine and orthophosphoric acid in a 2:1 molar ratio. The resulting mixture was stirred continuously for $3 \mathrm{~h}$ with constant heating maintained at $333 \mathrm{~K}$. The solution was then cooled, filtered and kept for crystallization without any disturbance. Good diffraction-quality crystals were obtained after one week.

\section{Refinement}

Crystal data, data collection and structure refinement details are summarized in Table 2. The hydrogen atoms associated with the $\mathrm{N}$ and $\mathrm{O}$ atoms were localized in a difference electron-density map and refined with the $\mathrm{N}-\mathrm{H}$ and $\mathrm{O}-\mathrm{H}$ distances constrained to values of 0.90 (2) and 0.85 (1) $\AA$, respectively. All other hydrogen atoms were placed in calculated positions and allowed to ride on their parent atoms, with $\mathrm{C}-\mathrm{H}=0.93 \AA$ and $U_{\text {iso }}(\mathrm{H})=1.2 U_{\text {eq }}(\mathrm{C})$.

\section{Acknowledgements}

The authors thank Dr Babu Varghese and SAIF, IIT Madras, India, for the data collection.

\section{References}

Balamurugan, P., Jagan, R. \& Sivakumar, K. (2010). Acta Cryst. C66, o109-o113.

Baouab, L. \& Jouini, A. (1998). J. Solid State Chem. 141, 343-351.

Bruker (2012). APEX2, SAINT, XPREP and $S A D A B S$. Bruker AXS Inc., Madison, Wisconsin, USA.

Direm, A., Altomare, A., Moliterni, A. \& Benali-Cherif, N. (2015). Acta Cryst. B71, 427-436.

Farrugia, L. J. (2012). J. Appl. Cryst. 45, 849-854.

Groom, C. R. \& Allen, F. H. (2014). Angew. Chem. Int. Ed. 53, 662671.

Ilioudis, C. A., Georganopoulou, D. G. \& Steed, J. W. (2002). CrystEngComm, 4, 26-36.

Jagan, R., Sathya, D. \& Sivakumar, K. (2015). Acta Cryst. C71, 374 380.

Khan, M. I., Nome, R. C., Deb, S., McNeely, J. H., Cage, B. \& Doedens, R. J. (2009). Cryst. Growth Des. 9, 2848-2852.

Li, X.-M., Feng, S.-S., Wang, F., Ma, Q. \& Zhu, M.-L. (2010). Acta Cryst. E66, o239-o240.

Macrae, C. F., Bruno, I. J., Chisholm, J. A., Edgington, P. R., McCabe, P., Pidcock, E., Rodriguez-Monge, L., Taylor, R., van de Streek, J. \& Wood, P. A. (2008). J. Appl. Cryst. 41, 466-470.

Mrad, M. L., Zeller, M., Hernandez, K. J., Rzaigui, M. \& Ben Nasr, C. (2012). Acta Cryst. E68, o3257-03258.

Oueslati, A., Kefi, R., Ben Nasr, C. \& Lefebvre, F. (2007). J. Mol. Struct. 871, 49-58.

Peng, R. \& Zhao, Y. (2010). Acta Cryst. E66, o3235.

Evans, I. R., Howard, J. A. K. \& Evans, J. S. O. (2008). Cryst. Growth Des. 8, 1635-1639.

Raghavaiah, P., Supriya, S. \& Das, S. K. (2005). CrystEngComm, 7, 167-170.

Rao, A. S., Tripuramallu, B. K., Ravada, K. \& Das, S. K. (2010). Acta Cryst. E66, o1945.

Sheldrick, G. M. (2008). Acta Cryst. A64, 112-122.

Sheldrick, G. M. (2015). Acta Cryst. C71, 3-8.

Shylaja, S., Mahendra, K. N., Varma, K. B. R., Narasimhamurthy, T. \& Rathore, R. S. (2008). Acta Cryst. C64, o361-o363.

Singh, U. P., Kashyap, S., Singh, H. J. \& Butcher, R. J. (2011). CrystEngComm, 13, 4110-4120.

Soumhi, E. H. \& Jouini, T. (1995). Acta Cryst. C51, 1883-1885.

Spek, A. L. (2009). Acta Cryst. D65, 148-155.

Trojette, B., Hajem, A. A., Driss, A. \& Jouini, T. (1998). Acta Cryst. C54, 1867-1869. 


\section{supporting information}

Acta Cryst. (2016). E72, 530-533 [https://doi.org/10.1107/S2056989016004709]

\section{Crystal structure of bis(2-aminoanilinium) hydrogen phosphate}

\section{Reena Ittyachan, Melesuparambil Sundaram Ahigna and Rajamony Jagan}

Computing details

Data collection: APEX2 (Bruker, 2012); cell refinement: APEX2 and SAINT (Bruker, 2012); data reduction: SAINT and XPREP (Bruker, 2012); program(s) used to solve structure: SHELXS2014 (Sheldrick, 2008); program(s) used to refine structure: SHELXL2014 (Sheldrick, 2015); molecular graphics: ORTEP-3 for Windows (Farrugia, 2012) and Mercury (Macrae et al., 2008); software used to prepare material for publication: PLATON (Spek, 2009).

Bis(2-aminoanilinium) hydrogen phosphate

Crystal data

$2 \mathrm{C}_{6} \mathrm{H}_{9} \mathrm{~N}_{2}+\cdot \mathrm{HPO}_{4}{ }^{2-}$

$M_{r}=314.28$

Triclinic, $P \overline{1}$

$a=4.7613(7) \AA$

$b=10.8925(17) \AA$

$c=15.054(2) \AA$

$\alpha=107.263(3)^{\circ}$

$\beta=94.060(3)^{\circ}$

$\gamma=94.549(3)^{\circ}$

$V=739.6(2) \AA^{3}$

Data collection

Bruker Kappa APEXII CCD Diffractometer $\omega$ and $\varphi$ scan

Absorption correction: multi-scan

(SADABS; Bruker, 2012)

$T_{\min }=0.865, T_{\max }=0.902$

16948 measured reflections

2841 independent reflections

Refinement

Refinement on $F^{2}$

Least-squares matrix: full

$R\left[F^{2}>2 \sigma\left(F^{2}\right)\right]=0.052$

$w R\left(F^{2}\right)=0.119$

$S=1.16$

2841 reflections

242 parameters

11 restraints
$Z=2$

$F(000)=332$

$D_{\mathrm{x}}=1.411 \mathrm{Mg} \mathrm{m}^{-3}$

Mo $K \alpha$ radiation, $\lambda=0.71073 \AA$

Cell parameters from 7191 reflections

$\theta=2.8-26.1^{\circ}$

$\mu=0.21 \mathrm{~mm}^{-1}$

$T=296 \mathrm{~K}$

Block, brown

$0.30 \times 0.20 \times 0.20 \mathrm{~mm}$

2271 reflections with $I>2 \sigma(I)$

$R_{\text {int }}=0.039$

$\theta_{\text {max }}=26.0^{\circ}, \theta_{\min }=2.0^{\circ}$

$h=-5 \rightarrow 5$

$k=-13 \rightarrow 13$

$l=-18 \rightarrow 18$

$\mathrm{H}$ atoms treated by a mixture of independent and constrained refinement

$w=1 /\left[\sigma^{2}\left(F_{\mathrm{o}}^{2}\right)+(0.0318 P)^{2}+0.8089 P\right]$

where $P=\left(F_{\mathrm{o}}^{2}+2 F_{\mathrm{c}}{ }^{2}\right) / 3$

$(\Delta / \sigma)_{\max }<0.001$

$\Delta \rho_{\max }=0.49 \mathrm{e} \AA^{-3}$

$\Delta \rho_{\min }=-0.34$ e $\AA^{-3}$ 


\section{Special details}

Geometry. All esds (except the esd in the dihedral angle between two 1.s. planes) are estimated using the full covariance matrix. The cell esds are taken into account individually in the estimation of esds in distances, angles and torsion angles; correlations between esds in cell parameters are only used when they are defined by crystal symmetry. An approximate (isotropic) treatment of cell esds is used for estimating esds involving l.s. planes.

Fractional atomic coordinates and isotropic or equivalent isotropic displacement parameters $\left(\hat{A}^{2}\right)$

\begin{tabular}{|c|c|c|c|c|}
\hline & $x$ & $y$ & $z$ & $U_{\text {iso }} * / U_{\text {eq }}$ \\
\hline $\mathrm{C} 1$ & $0.0716(5)$ & $0.9348(3)$ & 0.75677 (17) & $0.0294(6)$ \\
\hline $\mathrm{C} 2$ & $0.2315(7)$ & $0.8319(3)$ & $0.7246(2)$ & $0.0430(7)$ \\
\hline $\mathrm{H} 2$ & 0.3277 & 0.7991 & 0.7669 & $0.042(9)^{*}$ \\
\hline $\mathrm{C} 3$ & $0.2477(8)$ & 0.7789 (4) & $0.6308(2)$ & $0.0589(10)$ \\
\hline H3 & 0.3532 & 0.7097 & 0.6090 & $0.068(11)^{*}$ \\
\hline $\mathrm{C} 4$ & $0.1069(8)$ & $0.8290(4)$ & $0.5698(2)$ & $0.0618(10)$ \\
\hline $\mathrm{H} 4$ & 0.1203 & 0.7942 & 0.5061 & $0.082(13)^{*}$ \\
\hline $\mathrm{C} 5$ & $-0.0531(7)$ & 0.9289 (4) & $0.6000(2)$ & $0.0546(9)$ \\
\hline H5 & -0.1503 & 0.9597 & 0.5566 & $0.059(10)^{*}$ \\
\hline C6 & $-0.0730(6)$ & $0.9859(3)$ & 0.6957 (2) & $0.0375(7)$ \\
\hline N1 & $0.0743(5)$ & $0.9908(2)$ & $0.85708(15)$ & $0.0290(5)$ \\
\hline $\mathrm{H} 1 \mathrm{~A}$ & $0.238(5)$ & $1.046(3)$ & $0.881(2)$ & $0.060(10)^{*}$ \\
\hline H1B & $0.079(6)$ & $0.928(2)$ & $0.8862(19)$ & $0.039(8)^{*}$ \\
\hline $\mathrm{H} 1 \mathrm{C}$ & $-0.080(5)$ & $1.037(3)$ & $0.8734(19)$ & $0.040(8)^{*}$ \\
\hline $\mathrm{N} 2$ & $-0.2164(6)$ & $1.0939(3)$ & $0.7268(2)$ & $0.0492(7)$ \\
\hline $\mathrm{H} 2 \mathrm{~A}$ & $-0.300(7)$ & $1.106(3)$ & $0.7814(17)$ & $0.064(11)^{*}$ \\
\hline $\mathrm{H} 2 \mathrm{~B}$ & $-0.337(7)$ & $1.100(4)$ & $0.680(2)$ & $0.071(12)^{*}$ \\
\hline $\mathrm{C} 7$ & $0.6532(6)$ & $0.4711(3)$ & 0.79919 (19) & $0.0321(6)$ \\
\hline $\mathrm{C} 8$ & $0.7961(7)$ & $0.5283(3)$ & $0.7439(2)$ & $0.0493(8)$ \\
\hline $\mathrm{H} 8$ & 0.9487 & 0.5905 & 0.7699 & $0.049(9)^{*}$ \\
\hline C9 & $0.7133(9)$ & 0.4933 (4) & $0.6491(3)$ & $0.0683(11)$ \\
\hline H9 & 0.8085 & 0.5322 & 0.6110 & $0.076(12)^{*}$ \\
\hline $\mathrm{C} 10$ & $0.4896(9)$ & 0.4008 (4) & $0.6120(3)$ & $0.0694(12)$ \\
\hline H10 & 0.4328 & 0.3768 & 0.5483 & $0.071(11)^{*}$ \\
\hline C11 & $0.3490(8)$ & $0.3432(3)$ & $0.6677(2)$ & $0.0551(9)$ \\
\hline H11 & 0.1989 & 0.2799 & 0.6410 & $0.061(11)^{*}$ \\
\hline $\mathrm{C} 12$ & $0.4257(6)$ & $0.3773(3)$ & $0.7630(2)$ & $0.0371(7)$ \\
\hline N3 & $0.7285(5)$ & $0.5133(2)$ & $0.89942(16)$ & $0.0321(5)$ \\
\hline $\mathrm{H} 3 \mathrm{~A}$ & $0.895(5)$ & $0.564(3)$ & $0.917(2)$ & $0.052(10)^{*}$ \\
\hline H3B & $0.745(6)$ & 0.449 (2) & $0.9267(19)$ & $0.043(9)^{*}$ \\
\hline $\mathrm{H} 3 \mathrm{C}$ & $0.588(5)$ & $0.558(3)$ & $0.926(2)$ & $0.046(9)^{*}$ \\
\hline N4 & $0.2718(6)$ & $0.3266(3)$ & $0.8209(2)$ & $0.0508(7)$ \\
\hline $\mathrm{H} 4 \mathrm{~A}$ & $0.372(7)$ & $0.311(4)$ & $0.867(2)$ & $0.076(13)^{*}$ \\
\hline $\mathrm{H} 4 \mathrm{~B}$ & $0.135(6)$ & $0.266(3)$ & $0.790(2)$ & $0.065(11)^{*}$ \\
\hline $\mathrm{O} 1$ & $0.5217(4)$ & 0.8037 (2) & 0.92225 (14) & $0.0429(5)$ \\
\hline H1D & $0.692(3)$ & 0.799 (3) & $0.940(2)$ & $0.064(11)^{*}$ \\
\hline $\mathrm{O} 2$ & $0.0401(4)$ & $0.8199(2)$ & 0.95819 (16) & $0.0524(6)$ \\
\hline $\mathrm{O} 3$ & $0.2896(5)$ & $0.63962(19)$ & $0.98533(15)$ & $0.0475(6)$ \\
\hline $\mathrm{O} 4$ & $0.4165(4)$ & 0.86357 (19) & $1.08978(13)$ & $0.0407(5)$ \\
\hline
\end{tabular}




$\begin{array}{lllll}\mathrm{P} 1 & 0.31296(13) & 0.78084(6) & 0.99259(5) & 0.02434(19)\end{array}$

Atomic displacement parameters $\left(\AA^{2}\right)$

\begin{tabular}{lllllll}
\hline & $U^{11}$ & $U^{22}$ & $U^{33}$ & $U^{12}$ & $U^{13}$ & $U^{23}$ \\
\hline C1 & $0.0285(14)$ & $0.0316(15)$ & $0.0257(13)$ & $-0.0056(11)$ & $0.0010(11)$ & $0.0076(11)$ \\
C2 & $0.0474(18)$ & $0.0450(18)$ & $0.0349(16)$ & $0.0065(15)$ & $0.0069(14)$ & $0.0084(14)$ \\
C3 & $0.067(2)$ & $0.059(2)$ & $0.045(2)$ & $0.0181(19)$ & $0.0133(17)$ & $0.0015(17)$ \\
C4 & $0.065(2)$ & $0.080(3)$ & $0.0335(19)$ & $0.011(2)$ & $0.0065(16)$ & $0.0061(18)$ \\
C5 & $0.051(2)$ & $0.081(3)$ & $0.0347(17)$ & $0.0056(19)$ & $-0.0001(15)$ & $0.0248(18)$ \\
C6 & $0.0325(16)$ & $0.0440(17)$ & $0.0370(16)$ & $-0.0026(13)$ & $0.0014(12)$ & $0.0161(13)$ \\
N1 & $0.0293(13)$ & $0.0301(13)$ & $0.0267(12)$ & $0.0017(10)$ & $0.0011(10)$ & $0.0080(10)$ \\
N2 & $0.0471(17)$ & $0.0611(18)$ & $0.0478(17)$ & $0.0145(14)$ & $0.0051(14)$ & $0.0270(15)$ \\
C7 & $0.0321(15)$ & $0.0303(15)$ & $0.0350(15)$ & $0.0118(12)$ & $0.0032(12)$ & $0.0093(12)$ \\
C8 & $0.0430(19)$ & $0.059(2)$ & $0.051(2)$ & $0.0094(17)$ & $0.0126(15)$ & $0.0215(17)$ \\
C9 & $0.069(3)$ & $0.100(3)$ & $0.053(2)$ & $0.029(2)$ & $0.025(2)$ & $0.039(2)$ \\
C10 & $0.075(3)$ & $0.098(3)$ & $0.035(2)$ & $0.034(3)$ & $0.0019(19)$ & $0.015(2)$ \\
C11 & $0.060(2)$ & $0.053(2)$ & $0.0439(19)$ & $0.0135(18)$ & $-0.0113(17)$ & $0.0038(16)$ \\
C12 & $0.0409(17)$ & $0.0309(15)$ & $0.0377(16)$ & $0.0121(13)$ & $-0.0036(13)$ & $0.0075(12)$ \\
N3 & $0.0308(14)$ & $0.0290(13)$ & $0.0353(13)$ & $0.0021(11)$ & $0.0013(10)$ & $0.0083(11)$ \\
N4 & $0.0484(17)$ & $0.0438(17)$ & $0.0583(19)$ & $-0.0114(14)$ & $-0.0164(15)$ & $0.0215(15)$ \\
O1 & $0.0187(10)$ & $0.0748(16)$ & $0.0457(12)$ & $0.0028(10)$ & $0.0043(9)$ & $0.0346(11)$ \\
O2 & $0.0195(10)$ & $0.0860(18)$ & $0.0641(15)$ & $0.0096(10)$ & $0.0061(9)$ & $0.0404(13)$ \\
O3 & $0.0651(15)$ & $0.0264(11)$ & $0.0524(13)$ & $0.0050(10)$ & $0.0176(11)$ & $0.0115(9)$ \\
O4 & $0.0401(11)$ & $0.0419(12)$ & $0.0329(11)$ & $-0.0036(9)$ & $0.0010(9)$ & $0.0031(9)$ \\
P1 & $0.0160(3)$ & $0.0270(4)$ & $0.0319(4)$ & $0.0021(2)$ & $0.0026(2)$ & $0.0116(3)$ \\
& & & & & & \\
& & & & & &
\end{tabular}

Geometric parameters $\left(\hat{A},{ }^{\circ}\right)$

\begin{tabular}{llll}
\hline $\mathrm{C} 1-\mathrm{C} 6$ & $1.380(4)$ & $\mathrm{C} 8-\mathrm{C} 9$ & $1.382(5)$ \\
$\mathrm{C} 1-\mathrm{C} 2$ & $1.391(4)$ & $\mathrm{C} 8-\mathrm{H} 8$ & 0.9300 \\
$\mathrm{C} 1-\mathrm{N} 1$ & $1.450(3)$ & $\mathrm{C} 9-\mathrm{C} 10$ & $1.369(6)$ \\
$\mathrm{C} 2-\mathrm{C} 3$ & $1.368(4)$ & $\mathrm{C} 9-\mathrm{H} 9$ & 0.9300 \\
$\mathrm{C} 2-\mathrm{H} 2$ & 0.9300 & $\mathrm{C} 10-\mathrm{C} 11$ & $1.367(5)$ \\
$\mathrm{C} 3-\mathrm{C} 4$ & $1.363(5)$ & $\mathrm{C} 10-\mathrm{H} 10$ & 0.9300 \\
$\mathrm{C} 3-\mathrm{H} 3$ & 0.9300 & $\mathrm{C} 11-\mathrm{C} 12$ & $1.385(4)$ \\
$\mathrm{C} 4-\mathrm{C} 5$ & $1.363(5)$ & $\mathrm{C} 11-\mathrm{H} 11$ & 0.9300 \\
$\mathrm{C} 4-\mathrm{H} 4$ & 0.9300 & $\mathrm{C} 12-\mathrm{N} 4$ & $1.383(4)$ \\
$\mathrm{C} 5-\mathrm{C} 6$ & $1.403(4)$ & $\mathrm{N} 3-\mathrm{H} 3 \mathrm{~A}$ & $0.902(18)$ \\
$\mathrm{C} 5-\mathrm{H} 5$ & 0.9300 & $\mathrm{~N} 3-\mathrm{H} 3 \mathrm{~B}$ & $0.913(18)$ \\
$\mathrm{C} 6-\mathrm{N} 2$ & $1.384(4)$ & $\mathrm{N} 3-\mathrm{H} 3 \mathrm{C}$ & $0.906(18)$ \\
$\mathrm{N} 1-\mathrm{H} 1 \mathrm{~A}$ & $0.923(19)$ & $\mathrm{N} 4-\mathrm{H} 4 \mathrm{~A}$ & $0.880(19)$ \\
$\mathrm{N} 1-\mathrm{H} 1 \mathrm{~B}$ & $0.915(17)$ & $\mathrm{N} 4-\mathrm{H} 4 \mathrm{~B}$ & $0.885(19)$ \\
$\mathrm{N} 1-\mathrm{H} 1 \mathrm{C}$ & $0.931(17)$ & $\mathrm{O} 1-\mathrm{P} 1$ & $1.561(2)$ \\
$\mathrm{N} 2-\mathrm{H} 2 \mathrm{~A}$ & $0.919(18)$ & $\mathrm{O} 1-\mathrm{H} 1 \mathrm{D}$ & $0.846(10)$ \\
$\mathrm{N} 2-\mathrm{H} 2 \mathrm{~B}$ & $0.901(19)$ & $\mathrm{O} 2-\mathrm{P} 1$ & $1.504(2)$ \\
$\mathrm{C} 7-\mathrm{C} 8$ & $1.366(4)$ & $\mathrm{O} 3-\mathrm{P} 1$ & $1.504(2)$ \\
$\mathrm{C} 7-\mathrm{C} 12$ & $1.387(4)$ & $\mathrm{O} 4-\mathrm{P} 1$ & $1.497(2)$
\end{tabular}


C7-N3

$\mathrm{C} 6-\mathrm{C} 1-\mathrm{C} 2$

$\mathrm{C} 6-\mathrm{C} 1-\mathrm{N} 1$

$\mathrm{C} 2-\mathrm{C} 1-\mathrm{N} 1$

$\mathrm{C} 3-\mathrm{C} 2-\mathrm{C} 1$

$\mathrm{C} 3-\mathrm{C} 2-\mathrm{H} 2$

$\mathrm{C} 1-\mathrm{C} 2-\mathrm{H} 2$

$\mathrm{C} 4-\mathrm{C} 3-\mathrm{C} 2$

$\mathrm{C} 4-\mathrm{C} 3-\mathrm{H} 3$

$\mathrm{C} 2-\mathrm{C} 3-\mathrm{H} 3$

$\mathrm{C} 5-\mathrm{C} 4-\mathrm{C} 3$

$\mathrm{C} 5-\mathrm{C} 4-\mathrm{H} 4$

$\mathrm{C} 3-\mathrm{C} 4-\mathrm{H} 4$

$\mathrm{C} 4-\mathrm{C} 5-\mathrm{C} 6$

$\mathrm{C} 4-\mathrm{C} 5-\mathrm{H} 5$

$\mathrm{C} 6-\mathrm{C} 5-\mathrm{H} 5$

$\mathrm{C} 1-\mathrm{C} 6-\mathrm{N} 2$

$\mathrm{C} 1-\mathrm{C} 6-\mathrm{C} 5$

N2-C6-C5

$\mathrm{C} 1-\mathrm{N} 1-\mathrm{H} 1 \mathrm{~A}$

C1-N1-H1B

H1A-N1-H1B

$\mathrm{C} 1-\mathrm{N} 1-\mathrm{H} 1 \mathrm{C}$

$\mathrm{H} 1 \mathrm{~A}-\mathrm{N} 1-\mathrm{H} 1 \mathrm{C}$

H1B $-\mathrm{N} 1-\mathrm{H} 1 \mathrm{C}$

$\mathrm{C} 6-\mathrm{N} 2-\mathrm{H} 2 \mathrm{~A}$

$\mathrm{C} 6-\mathrm{N} 2-\mathrm{H} 2 \mathrm{~B}$

$\mathrm{H} 2 \mathrm{~A}-\mathrm{N} 2-\mathrm{H} 2 \mathrm{~B}$

$\mathrm{C} 8-\mathrm{C} 7-\mathrm{C} 12$

$\mathrm{C} 8-\mathrm{C} 7-\mathrm{N} 3$

$\mathrm{C} 12-\mathrm{C} 7-\mathrm{N} 3$

C7-C8-C9

$\mathrm{C} 6-\mathrm{C} 1-\mathrm{C} 2-\mathrm{C} 3$

$\mathrm{N} 1-\mathrm{C} 1-\mathrm{C} 2-\mathrm{C} 3$

$\mathrm{C} 1-\mathrm{C} 2-\mathrm{C} 3-\mathrm{C} 4$

$\mathrm{C} 2-\mathrm{C} 3-\mathrm{C} 4-\mathrm{C} 5$

$\mathrm{C} 3-\mathrm{C} 4-\mathrm{C} 5-\mathrm{C} 6$

$\mathrm{C} 2-\mathrm{C} 1-\mathrm{C} 6-\mathrm{N} 2$

$\mathrm{N} 1-\mathrm{C} 1-\mathrm{C} 6-\mathrm{N} 2$

$\mathrm{C} 2-\mathrm{C} 1-\mathrm{C} 6-\mathrm{C} 5$

$\mathrm{N} 1-\mathrm{C} 1-\mathrm{C} 6-\mathrm{C} 5$

$\mathrm{C} 4-\mathrm{C} 5-\mathrm{C} 6-\mathrm{C} 1$

$\mathrm{C} 4-\mathrm{C} 5-\mathrm{C} 6-\mathrm{N} 2$
$1.450(4)$

$121.3(3)$

$121.2(2)$

$117.5(2)$

$120.2(3)$

119.9

119.9

119.0 (3)

120.5

120.5

$121.6(3)$

119.2

119.2

120.8 (3)

119.6

119.6

121.9 (3)

$117.1(3)$

$120.8(3)$

$110(2)$

110.7 (19)

105 (3)

112.3 (18)

109 (3)

$110(3)$

$118(2)$

$110(2)$

$112(3)$

$121.9(3)$

$119.8(3)$

$118.2(3)$

$119.8(4)$

$-0.1(5)$

$-177.0(3)$

$0.5(5)$

$-1.1(6)$

$1.5(6)$

$-175.3(3)$

$1.4(4)$

$0.4(4)$

$177.2(3)$

$-1.1(5)$

$174.7(3)$

$\begin{array}{ll}\mathrm{C} 7-\mathrm{C} 8-\mathrm{H} 8 & 120.1 \\ \mathrm{C} 9-\mathrm{C} 8-\mathrm{H} 8 & 120.1 \\ \mathrm{C} 10-\mathrm{C} 9-\mathrm{C} 8 & 119.3(4) \\ \mathrm{C} 10-\mathrm{C} 9-\mathrm{H} 9 & 120.4 \\ \mathrm{C} 8-\mathrm{C} 9-\mathrm{H} 9 & 120.4 \\ \mathrm{C} 11-\mathrm{C} 10-\mathrm{C} 9 & 120.6(3) \\ \mathrm{C} 11-\mathrm{C} 10-\mathrm{H} 10 & 119.7 \\ \mathrm{C} 9-\mathrm{C} 10-\mathrm{H} 10 & 119.7 \\ \mathrm{C} 10-\mathrm{C} 11-\mathrm{C} 12 & 121.3(4) \\ \mathrm{C} 10-\mathrm{C} 11-\mathrm{H} 11 & 119.3 \\ \mathrm{C} 12-\mathrm{C} 11-\mathrm{H} 11 & 119.3 \\ \mathrm{~N} 4-\mathrm{C} 12-\mathrm{C} 11 & 121.7(3) \\ \mathrm{N} 4-\mathrm{C} 12-\mathrm{C} 7 & 121.0(3) \\ \mathrm{C} 11-\mathrm{C} 12-\mathrm{C} 7 & 117.1(3) \\ \mathrm{C} 7-\mathrm{N} 3-\mathrm{H} 3 \mathrm{~A} & 113(2) \\ \mathrm{C} 7-\mathrm{N} 3-\mathrm{H} 3 \mathrm{~B} & 116.0(19) \\ \mathrm{H} 3 \mathrm{~A}-\mathrm{N} 3-\mathrm{H} 3 \mathrm{~B} & 105(3) \\ \mathrm{C} 7-\mathrm{N} 3-\mathrm{H} 3 \mathrm{C} & 107(2) \\ \mathrm{H} 3 \mathrm{~A}-\mathrm{N} 3-\mathrm{H} 3 \mathrm{C} & 110(3) \\ \mathrm{H} 3 \mathrm{~B}-\mathrm{N} 3-\mathrm{H} 3 \mathrm{C} & 105(3) \\ \mathrm{C} 12-\mathrm{N} 4-\mathrm{H} 4 \mathrm{~A} & 116(3) \\ \mathrm{C} 12-\mathrm{N} 4-\mathrm{H} 4 \mathrm{~B} & 113(2) \\ \mathrm{H} 4 \mathrm{~A}-\mathrm{N} 4-\mathrm{H} 4 \mathrm{~B} & 116(4) \\ \mathrm{P} 1-\mathrm{O} 1-\mathrm{H} 1 \mathrm{D} & 113(2) \\ \text { O4-P1-O2 } & 111.69(13) \\ \text { O4-P1-O3 } & 111.37(12) \\ \text { O2-P1-O3 } & 111.93(14) \\ \text { O4-P1-O1 } & 110.20(12) \\ \text { O2-P1-O1 } & 103.14(11) \\ \text { O3-P1-O1 } & 108.14(12) \\ & \end{array}$

$\mathrm{C} 12-\mathrm{C} 7-\mathrm{C} 8-\mathrm{C} 9 \quad 0.4(5)$

N3-C7-C8-C9 -176.2 (3)

$\mathrm{C} 7-\mathrm{C} 8-\mathrm{C} 9-\mathrm{C} 10 \quad-0.5(5)$

$\mathrm{C} 8-\mathrm{C} 9-\mathrm{C} 10-\mathrm{C} 11 \quad 0.0(6)$

$\mathrm{C} 9-\mathrm{C} 10-\mathrm{C} 11-\mathrm{C} 12 \quad 0.7(6)$

$\mathrm{C} 10-\mathrm{C} 11-\mathrm{C} 12-\mathrm{N} 4 \quad 175.2(3)$

$\mathrm{C} 10-\mathrm{C} 11-\mathrm{C} 12-\mathrm{C} 7 \quad-0.8(5)$

$\mathrm{C} 8-\mathrm{C} 7-\mathrm{C} 12-\mathrm{N} 4 \quad-175.7$ (3)

$\mathrm{N} 3-\mathrm{C} 7-\mathrm{C} 12-\mathrm{N} 4 \quad 0.9(4)$

$\mathrm{C} 8-\mathrm{C} 7-\mathrm{C} 12-\mathrm{C} 11 \quad 0.3(4)$

N3-C7-C12-C11 176.9 (2) 
supporting information

Hydrogen-bond geometry $\left(\AA,{ }^{\circ}\right)$

\begin{tabular}{lllll}
\hline$D-\mathrm{H} \cdots A$ & $D-\mathrm{H}$ & $\mathrm{H} \cdots A$ & $D \cdots A$ & $D-\mathrm{H}^{\cdots} A$ \\
\hline $\mathrm{O} 1-\mathrm{H} 1 D \cdots \mathrm{O} 2^{\mathrm{i}}$ & $0.85(1)$ & $1.65(1)$ & $2.470(3)$ & $164(4)$ \\
$\mathrm{N} 3-\mathrm{H} 3 A \cdots 3^{\mathrm{i}}$ & $0.90(2)$ & $2.06(2)$ & $2.928(3)$ & $160(3)$ \\
$\mathrm{N} 1-\mathrm{H} 1 A \cdots 4^{\text {ii }}$ & $0.92(2)$ & $1.81(2)$ & $2.720(3)$ & $171(3)$ \\
$\mathrm{N} 1-\mathrm{H} 1 C \cdots \mathrm{O} 4^{i i i}$ & $0.93(2)$ & $2.02(2)$ & $2.953(3)$ & $179(3)$ \\
$\mathrm{N} 2-\mathrm{H} 2 A \cdots \mathrm{O} 4^{\text {iii }}$ & $0.92(2)$ & $1.99(2)$ & $2.904(4)$ & $170(3)$ \\
$\mathrm{N} 4-\mathrm{H} 4 A \cdots \mathrm{O} 44^{\text {iv }}$ & $0.88(2)$ & $2.45(3)$ & $3.188(4)$ & $142(3)$ \\
$\mathrm{N} 3-\mathrm{H} 3 B \cdots \mathrm{O} 3^{\text {iv }}$ & $0.91(2)$ & $1.87(2)$ & $2.740(3)$ & $159(3)$ \\
$\mathrm{N} 3-\mathrm{H} 3 C \cdots \mathrm{O} 3$ & $0.91(2)$ & $1.87(2)$ & $2.778(3)$ & $176(3)$ \\
$\mathrm{N} 1-\mathrm{H} 1 B \cdots \mathrm{O} 2$ & $0.92(2)$ & $1.83(2)$ & $2.734(3)$ & $169(3)$ \\
$\mathrm{N} 4-\mathrm{H} 4 B \cdots \mathrm{N} 2^{v}$ & $0.89(2)$ & $2.33(2)$ & $3.210(4)$ & $172(3)$
\end{tabular}

Symmetry codes: (i) $x+1, y, z$; (ii) $-x+1,-y+2,-z+2$; (iii) $-x,-y+2,-z+2$; (iv) $-x+1,-y+1,-z+2$; (v) $x, y-1, z$. 